Archives

14-15 | 1995

Les banques de données au $\mathrm{CRH}$

\title{
Les banques de données au CRH - présentation
}

\section{Patrice Bourdelais}

\section{(2) OpenEdition}

\section{Journals}

Édition électronique

URL : http://journals.openedition.org/ccrh/2657

DOI : $10.4000 /$ ccrh. 2657

ISSN : $1760-7906$

Éditeur

Centre de recherches historiques - EHESS

Édition imprimée

Date de publication : 14 octobre 1995

ISSN : 0990-9141

Référence électronique

Patrice Bourdelais, "Les banques de données au CRH - présentation », Les Cahiers du Centre de Recherches Historiques [En ligne], 14-15 | 1995, mis en ligne le 20 avril 2009, consulté le 10 décembre 2020. URL : http://journals.openedition.org/ccrh/2657 ; DOI : https://doi.org/10.4000/ccrh.2657

Ce document a été généré automatiquement le 10 décembre 2020.

Article L.111-1 du Code de la propriété intellectuelle. 


\title{
Les banques de données au CRH - présentation
}

\author{
Patrice Bourdelais
}

1 La volonté et l'obstination peuvent parfois renverser les montagnes. En dépit de l'absence d'une assistance informatique structurée et de moyens spécifiques en crédits comme en personnel, les chercheurs du CRH ont développé, depuis près de vingt ans, la constitution de banques de données ${ }^{1}$. Sur les 19 entreprises en cours recensées, 11 sont présentées dans les pages qui suivent. Elles concernent aussi bien l'histoire médiévale que la période contemporaine, l'histoire rurale que le monde urbain, l'histoire démographique et sociale que l'approche culturelle. Elles sont de taille variable et se situent à des degrés d'avancement très différents. Elles retracent à leur manière, partielle, l'évolution de la recherche au CRH.

2 Même si une telle distinction est partiellement erronée, nous présentons d'abord les banques de données factuelles, préoccupées uniquement de recherche, puis les banques de données documentaires dont le but est avant tout de constituer et de diffuser des instruments de travail, même si leurs promoteurs sont bien légitimement tentés d'effectuer des premiers traitements grâce à l'outil qu'ils viennent de construire.

Opération pionnière, le catasto florentin de 1427 a été saisi et traité entre 1966 et 1976, ses résultats ont été publiés dès 1978 dans un ouvrage de référence ${ }^{2}$. Christiane KlapischZuber rappelle aujourd'hui la richesse du document et les objectifs de «sa mise en machine", elle souligne ses développements possibles car non seulement une telle description a été répétée, mais on pourrait l'enrichir par l'adjonction d'autres types de sources afin de coupler les données réordonnées nominativement et opérer des approches longitudinales. Celles-ci constituent la nouvelle orientation majeure de la recherche en histoire démographique et sociale depuis plus de dix ans, elles ont été mises en oeuvre aussi bien par l'enquête des 3000 familles (J. Dupâquier) que par l'étude des itinéraires individuels d'une population de l'industrie, celle du Creusot (P. Bourdelais et M. Demonet). Les banques de données sont ici les outils de la reconstitution de généalogies ou d'itinéraires individuels et familiaux, elles constituent aussi la possibilité 
de restituer des processus dans leur complexité, de tester des hypothèses fines sur des ensembles numériquement significatifs.

4 Moins quantitatives, les banques de données constituées à partir des inventaires après décès (F. Piponnier, "Inventaires bourguignons $\mathrm{XIV}^{\mathrm{e}}-\mathrm{XV}^{\mathrm{e}}$ siècles »; M. Baulant, «Les inventaires mobiliers de la région de Meaux») ont également pour but de traiter d'énormes masses documentaires, d'en faire émerger de nouvelles connaissances lexicologiques, d'enrichir l'histoire de la consommation et celle de la vie matérielle, par la prise en compte des objets éphémères et périssables en complément des traditionnels inventaires archéologiques.

5 Ces banques de données sont prioritairement des fichiers de travail, organisés en fonction d'une problématique donnée, afin d'aboutir à des connaissances inédites, tant par de nouveaux apports descriptifs que par la possibilité de tester de nouvelles hypothèses. Mais à aucun moment leur publication ou leur diffusion n'a été envisagée car il ne s'agit pas d'instruments de travail dont la consultation serait directement utile à l'ensemble des chercheurs. En revanche, elles sont créatrices de véritables coopérations internationales fondées sur l'exploitation de fichiers semblables suivant des méthodologies négociées et partagées, conditions de faisabilité d'une histoire comparée raisonnée.

La catégorie des banques de données documentaires n'est pourtant pas absente du CRH. Plusieurs d'entre elles, parmi les pionnières, concernent l'époque médiévale. Il s'agit alors de repérer, d'indexer, de décrire afin de rendre accessibles des textes ("Exempla ", M.-A. Polo de Beaulieu) ou des images qui ne l'étaient pas (P. Mane, «Une banque de données d'images »; J. Baschet, «Les bases de données du groupe de recherches sur les images médiévales »). Ces opérations valent non seulement par la qualité de la recherche documentaire, effectuée dans de nombreux lieux, qui alimente la base, mais aussi par la pertinence des mots-clés retenus et celle de l'indexation. Le produit fini, par exemple les vidéodisques de la Bibliothèque vaticane, devient alors un prodigieux instrument de travail qui permet à celui qui l'utilise d'effectuer en quelques heures un dépouillement qui demandait plusieurs années il y a bien peu de temps encore.

7 La création d'un corpus informatisé de la littérature agricole imprimée en Europe au XVI ${ }^{\mathrm{e}}$ siècle (C. Beutler) rejoint, par son double objectif (mettre à la disposition des chercheurs un outil de travail, mais aussi établir la constitution, l'évolution et la diffusion de ces savoirs), la grande enquête lancée par P. Jeannin et J. Hoock sur les manuels et traités à l'usage des marchands (1470-1820). Ars Mercatoria propose un inventaire des livres imprimés en Europe correspondant à une fonction : l'usage du marchand. Le corpus est celui des ouvrages conservés dans les grandes bibliothèques, il permet de préciser les disciplines popularisées par ces publications, d'analyser leur style ainsi que les directions de leur diffusion et leur chronologie. Cette double vocation se retrouve dans la banque de données MEDOC (mécanisation de la France $\mathrm{XIX}^{\mathrm{e}}$-XX $\mathrm{XX}^{\mathrm{e}}$ siècles) présentée ici par $\mathrm{S}$. Benoit et G. Emptoz. L'informatisation de plusieurs dépouillements manuels relatifs au moteur hydraulique, aux industries de la région parisienne, aux brevets déposés dans le domaine de la cokéfaction, ont permis de reconstituer les fichiers des inventeurs, des acteurs de l'innovation, entrepreneurs et entreprises. L'adjonction des renseignements issus d'archives d'entreprises, en particulier de construction de machines textiles, conduit à l'analyse des débouchés, par conséquent des entreprises clientes en France et à l'étranger. En reliant ces fichiers les uns aux autres, les approches croisées à partir d'observatoires différents se multiplient, ce qui n'était pas possible jusqu'alors. 
8 Non seulement les banques de données permettent d'organiser les vastes corpus disponibles, d'en construire de nouveaux, de dominer et de rendre ainsi accessibles de très vastes ensembles documentaires, mais elles permettent aussi d'effectuer des recoupements entre fichiers auparavant distincts, de reconstituer des histoires individuelles longues et riches, de procéder à des analyses qui restaient auparavant hors de portée de l'historien. Elles constituent aussi d'indiscutables outils de rayonnement international. A ce titre au moins, elles mériteraient davantage d'attention de la part des pouvoirs publics qui gèrent les ressources budgétaires et les postes destinés à la recherche dans notre pays.

\section{NOTES}

1. A titre d'élément de comparaison, signalons que la DDB (Demographic Data Base) suédoise, répartie et dirigée depuis l'université d'Umea, emploie environ soixante personnes et dispose de plusieurs dizaines de millions de francs de crédits annuels.

2. D. Herlihy et C. Klapisch-Zuber, Les Toscans et leurs familles. Une étude du catasto florentin de 1427, Paris, Éditions de l'EHESS et Presses de la FNSP, 1978. 